\title{
Elaboración y Validación de un Instrumento para la Medición de la Violencia Laboral Externa y sus Factores de Riesgo en Población de Trabajadores y Trabajadoras Chileno/as \\ DEVELOPMENT AND VALIDATION OF AN INSTRUMENT FOR THE MEASUREMENT OF EXTERNAL VIOLENCE AT WORK AND ITS RISK FACTORS IN A POPULATION OF CHILEAN WORKERS
}

Ximena Díaz Berr1, Amalia Mauro Cardarelli², Cristián Villarroel Poblete ${ }^{3}$, Juan Pablo Toro Cifuentes ${ }^{4}$, Daniela Campos Schwarze ${ }^{5}$

1. Socióloga, investigadora Centro de Estudios de la Mujer, Santiago, Chile

2. Socióloga. Investigadora, Directora, Centro de Estudios de la Mujer, Santiago, Chile

3. Sociólogo, Departamento Salud Ocupacional, Ministerio de Salud, Santiago Chile

4. Psicólogo, Académico Programa de Estudios Psicosociales del Trabajo, Universidad Diego Portales. Santiago, Chile

5. Psicóloga, Asociación Chilena de Seguridad, Santiago, Chile

\begin{abstract}
RESUMEN
La violencia laboral externa, aquella que ejercen personas que no tienen una relación de trabajo directa con las víctimas (clientes, usuarios, asaltantes), es parte constitutiva de la violencia laboral, un problema de salud pública de creciente magnitud. En Chile la medición de la violencia laboral externa así como sus vínculos con la salud mental han sido poco estudiados, en parte por la inexistencia de instrumentos de evaluación. Objetivo: Elaborar y validar un instrumento para medir y caracterizar violencia externa, factores de riesgo y efectos en salud mental en trabajadores/as de la salud, educación, retail y administración pública. Metodología: técnicas cualitativas y cuantitativas: grupos focales, panel de expertos y aplicación del instrumento a 554 trabajadores/as. Resultados: El análisis factorial obtiene 7 factores referidos a violencia física, no fisica y a causas vinculadas al lugar de trabajo, los/ as usuarios/as y los/as trabajadores/as. La consistencia interna y confiabilidad del instrumento son estadísticamente significativas. Se confirma su capacidad para discriminar la existencia de violencia laboral externa, su intensidad y factores de riesgo en los sectores estudiados. No se observan correlaciones significativas con psicopatología medida a través del GHQ-12. Conclusiones: El instrumento tiene las propiedades para discriminar y medir la violencia laboral externa en los sectores estudiados.
\end{abstract}

(Díaz X, Mauro A, Villarroel C, Toro J, Campos D, 2018. Elaboración y Validación de un Instrumento para la Medición de la Violencia Laboral Externa y sus Factores de Riesgo en Población de Trabajadores y Trabajadoras Chileno/as. Cienc Trab. May-Ago; 20 [62]: 61-69).

Palabras claves: VIOLENCIA EXTERNA, VALIDACIÓN INSTRUMENTO, SALUD MENTAL

\section{ABSTRACT}

External workplace violence, is understood as that perpetrated by persons outside the organizations such us customers, users, assailants, and it is a form of workplace violence, a problem of public health of growing magnitude. In Chile, the measurement of external labor violence as well as its links with mental health have been little studied, in part by the absence of evaluation tools. Objective: to develop and validate an instrument to measure and characterize external workplace violence, risk factors and effects on mental health in workers from the health, education, retail, and public administration. Methodology: qualitative and quantitative techniques: focus groups, expert panel and application of the instrument to 554 workers. Results: factor analysis obtained 7 factors relating to physical, not physical violence and causes related to the workplace, the users, and workers - men and women. The internal consistency and reliability of the instrument are statistically significant. Confirms its ability to discriminate the existence of external workplace violence, it intensity and risk factors in the studied sectors. Failure to observe significant correlations with psychopathology measured by GHQ-12. Conclusion: The instrument has the properties to discriminate and measure the external workplace violence in the sectors considered.

Key words:

\section{INTRODUCCIÓN}

Se ha reconocido internacionalmente que la violencia y el acoso psicológico en el trabajo constituyen uno de los problemas de

Correspondencia / Correspondence:

Ximena Diaz Berr,

Paseo Bulnes 120, oficina 88,

Santiago, Chile

Tel: 226719418

e mail:ximenadb@gmail.com

Recibido: 29 de Mayo de 2018 / Aceptado: 14 de Julio de 2018

salud pública que mayor preocupación provocan en el mundo por su creciente magnitud. ${ }^{1-4}$

Gran parte de los estudios sobre el tema se han focalizado en identificar las diversas formas de violencia que se dan al interior de los lugares de trabajo, esto es, entre personas que desempeñan sus actividades dentro de una organización, institución o empresa. 
Estos estudios han entregado antecedentes relativos a las personas o grupos más vulnerables a la violencia, a los factores facilitadores o promotores de violencia, a formas de enfrentamiento de parte de las víctimas y a sus efectos para las personas, los colectivos laborales y las organizaciones / empresas, entre otros temas abordados. Si bien los agentes agresores o instigadores de violencia pueden encontrarse al interior de las organizaciones -compañeros, superiores o subordinados-, numerosos estudios han mostrado que en determinadas actividades laborales los y las trabajadoras están sometidas a riesgos que pueden afectar su seguridad y salud y que tienen un origen externo a la empresa, organización o institución. Se trata en este caso de la violencia externa, definida como aquella ejercida contra un/a trabajador/a por terceras personas, esto es, por personas sin relación profesional directa con la víctima, en el lugar de trabajo. Esta puede ser a la vez de dos tipos. La violencia tipo I que se caracteriza porque quienes llevan a cabo las acciones violentas no tienen ninguna relación legítima de trato con la víctima. Es decir, no existe un trato comercial o de usuario entre el agresor y la víctima (como asaltos y robos). La violencia tipo II se caracteriza porque en estos casos existe algún tipo de relación profesional entre el/la causante del acto violento y la actividad profesional del/la trabajador/a y se producen habitualmente mientras hay un intercambio de bienes y servicios. En contraste con los de tipo I, en las que su periodicidad y ocurrencia es irregular en la vida de un determinado lugar de trabajo, los de tipo II surgen del contacto habitual y diario de los trabajadores y trabajadoras con usuarios. $^{\text {5-10 }}$

En Chile, la problemática de la violencia externa se ha instalado en la discusión sobre las condiciones de trabajo. Prueba de ello es el tratamiento que recibió en los "Coloquios de Salud Ocupacional 2014” organizados por el Departamento de Salud Ocupacional del Ministerio de Salud, donde una de las problemáticas abordadas desde el mundo sindical y académico fue la "Preocupación por la violencia de los pacientes hacia trabajadores y trabajadoras en el Sector Salud", esto es, violencia generada por los usuarios del servicio. La preocupación por este problema radica en la ausencia de un protocolo de pesquisa de factores de riesgo asociados a la prevalencia de violencia externa que permita promover acciones o actividades de prevención y, por otra parte, cambios en las condiciones de seguridad de los ambientes laborales.

No obstante, el abordaje de este problema se ha visto limitado por la escasa información disponible en el país sobre esta materia. Algunos estudios realizados sobre violencia externa se han focalizado en la violencia tipo II $^{11-13}$ entregando evidencias de que este es un problema que viven cotidianamente las personas que desempeñan trabajos que implican atención a público. A nivel internacional, la encuesta Eqcotesst ${ }^{7}$ en Canadá, y los informes Eurofound ${ }^{14,7}$ sobre la situación en países europeos revelan resultados similares. El sector de la salud ha sido uno de los sectores más estudiados por los altos niveles de violencia externa a los que están expuestos los/as trabajadores/as del sector. En Chile se destaca el estudio de Paravic et al. ${ }^{11} \mathrm{y}$ a nivel internacional estudios recopilados por Cooper y Swanson ${ }^{15} \mathrm{e}$ informes divulgados por la European Agency for Safety and Health at Work. ${ }^{9,16}$

Los enfoques explicativos de la violencia externa señalan que esta puede ser resultado de interacciones interpersonales negativas, las que, a su vez, están insertas en un contexto organizacional y social más amplio en el cual se desarrollan. Se ha puesto particular atención a algunos factores que podrían influir en la naturaleza de los intercambios entre las partes que interactúan. Tales factores se extienden desde características de los sujetos involucrados, hasta el ambiente y el contexto socio cultural en el cual estas tienen lugar. Por otra parte, autoras como Billing ${ }^{17}$ y Krieger ${ }^{18}$ señalan la necesidad de considerar en los estudios sobre violencia laboral, las discriminaciones sociales y de género predominantes en la sociedad en general, y que se reproducen en las prácticas y sistemas de interacciones cotidianas.

En esta perspectiva, el foco de la explicación de la agresión y violencia debe ponerse en un análisis situacional y no solo en la relación agresor/víctima. En otras palabras, la violencia externa en el lugar de trabajo no es un problema individual ni aislado, sino estructural y estratégico con raíces en factores sociales, económicos, organizacionales y culturales. ${ }^{10,19}$ Como punto de partida, se recomienda analizar las situaciones potencialmente agresivas que se producen en los centros de trabajo para identificar los riesgos. ${ }^{20}$ En esta perspectiva, se ha señalado que hay ciertos procesos, situaciones e interacciones inherentes al trabajo que exponen a los/as trabajadores/as a un mayor riesgo de sufrir violencia externa en el lugar de trabajo.

En el contexto de las políticas públicas sobre riesgos psicosociales en Chile, se ha planteado la necesidad de incluir los riesgos psicosociales asociados a violencia externa y disponer de información respecto a la situación actual de trabajadores y trabajadoras en relación con este problema así como a los efectos en su salud mental, a fin de elaborar un protocolo de pesquisa de factores de riesgo que permitan promover acciones, actividades de prevención y de cambio en las condiciones de seguridad de los ambientes laborales.

Con todos estos antecedentes, este proyecto se propuso como objetivo elaborar y validar un instrumento para medir y caracterizar la violencia externa de la que son víctimas trabajadores/as de la salud, educación, retail y administración pública, identificar sus factores de riesgo y estudiar los efectos en su salud mental, a fin de contribuir al diseño de acciones y medidas de prevención.

\section{MÉTODO}

\section{Diseńo, Validación y Estandarización del Instrumento}

El proceso de validación y estandarización del instrumento "Encuesta sobre Violencia externa en el lugar de trabajo" se realizó mediante técnicas cualitativas y cuantitativas, y se desarrolló en cuatro etapas que se describen a continuación.

\section{Primera etapa: Elaboración del instrumento}

En consideración de que no se contaba con un instrumento específico para medir violencia externa, se elaboró una primera versión en base a la revisión bibliográfica sobre el tema y a la realización de grupos focales. Se realizaron 7 grupos focales con participación de 34 trabajadores y trabajadoras de los cuatro sectores económicos seleccionados, cuyas ocupaciones les exige la atención a distintos tipos de público. La información obtenida en los grupos focales permitió identificar un conjunto de situaciones de violencia externa que enfrentan en sus trabajos, así como las personas que las producen y los factores que a su juicio facilitan su ocurrencia. En base a esta información se elaboró la primera versión del instrumento.

A continuación se realizó la validación de contenidos de dicho instrumento por parte de un grupo de 8 expertos/as en el tema. A partir de los comentarios recogidos, se reformuló el instrumento dando lugar a la segunda versión del mismo. 
La adaptación semántica de esta segunda versión del instrumento se realizó mediante su aplicación a un total de 46 trabajadores/as del sector de la salud (profesionales y no profesionales), del sector del retail (cajeras/os, trabajadores/as de venta, guardias) y en educación a docentes de educación básica y media de establecimientos públicos y subvencionados. Sobre la base de la información recogida se introdujeron diversos cambios al instrumento que concluyeron con la elaboración de la tercera versión del mismo.

\section{Segunda etapa: Análisis factorial exploratorio}

A fin de estudiar las propiedades psicométricas del instrumento, se llevó a cabo un análisis factorial exploratorio en base a la aplicación de la encuesta a una muestra de 189 casos. Este estudio estuvo destinado a: a) Evaluar la variabilidad interna de cada una de las preguntas o reactivos, b) realizar un análisis con todos los items para determinar el grado de consistencia entre las preguntas que forman parte del instrumento, y c) colapsar preliminarmente los factores en sus medias.

Se utilizó un valor de eigen value mayor a 1 como criterio para determinar el número de factores. Una solución de cinco factores fue arrojada tras éste. Asimismo, se tomó un criterio conservador de 0.7 para determinar la inclusión de ítems en cada dimensión. Ninguno de ellos debió ser suprimido.

Los factores presentan altos niveles bajo la prueba de alpha de Cronbach, todos superiores a .898. Dadas las poco significativas diferencias en la confiabilidad total al retirar cada ítem, no se recomendó la eliminación de reactivos.

En base a estos resultados la tercera versión del instrumento no se modificó.

Tercera etapa: Análisis psicométrico y estandarización del instrumento

El análisis psicométrico y la estandarización del instrumento se realizaron con el total de la muestra (554 casos). El primero incluyó (a) Análisis factorial y (b) Pruebas de validez convergente: análisis de correlaciones entre los distintos factores y la medida de salud (GHQ-12). El segundo corresponde al cálculo de puntajes, punto de corte o baremos sobre el grado de exposición a violencia externa.

\section{Descripción del instrumento definitivo}

El instrumento elaborado consta de 4 partes. La primera está constituida por dos escalas. Una compuesta por 14 reactivos que plantean una aseveración o afirmación sobre alguna situación o comportamiento constituyente de maltrato o violencia laboral por parte de clientes/usuarios. La segunda compuesta por 4 reactivos que describen situaciones de violencia ejercida por personas que no tienen una relación legítima de trato con las víctimas, es decir delincuentes o asaltantes. En ambas escalas se solicita al trabajador/a que señale las conductas de violencia que ha experimentado en su trabajo y también la frecuencia de las mismas. Se proporcionan cuatro opciones de respuesta (de 1=nunca a $4=$ siempre). Estas respuestas permiten contar con información sobre la presencia de conductas que se consideran maltrato o violencia laboral externa y sobre su intensidad. Se pide también señalar quiénes son las personas que ejercen violencia (clientes, pacientes, usuarios, estudiantes, entre otros) y el sexo de las mismas.

En la segunda parte se pregunta por las causas o los factores que posibilitan el desencadenamiento de maltrato por parte de personas ajenas a la empresa o institución y la frecuencia con que ocurren. Se proporcionan cuatro opciones de respuesta (de $1=$ nunca a 4 =siempre). Estos factores se subdividieron en 3 categorías: Factores relacionados con el lugar de trabajo (empresa o institución), factores relacionados con el usuario y factores relacionados con el/la trabajador/a.

En la tercera parte se solicita a los/as encuestados/as responder al GHQ-12 sobre percepción de salud general. Por último, en la cuarta parte, se les solicita información general, sociodemográfica y laboral: sexo, edad, nivel de educación, puesto o cargo que ocupa, antigüedad en la empresa/institución, horario de trabajo, tipo de contrato y de jornada.

\section{Diseńo Muestral}

\section{i. Población objetivo}

Se definió como población objetivo a los/as trabajadores/as asalariados/as de las empresas adheridas a la ACHS, pertenecientes a cuatro sectores de la economía: comercio, salud, educación y administración pública, de la Región Metropolitana, y que se relacionan con público. En el comercio, vendedores/as, cajeros/as, guardias; en salud, trabajadores no profesionales y profesionales no médicos en atención a público; en educación, docentes de enseñanza básica y media y en administración pública, personas que atienden público.

\section{ii. Diseño muestral}

El diseño de la muestra consistió en primer lugar en seleccionar -de las empresas adheridas a la Asociación Chilena de Seguridad en la región metropolitana- una empresa al azar por cada sector estudiado en cada zona geográfica (Norte, Sur, Oriente, Poniente). Es decir, la muestra quedaría compuesta por 16 empresas: 4 por cada zona geográfica y en cada zona se incluía una empresa por sector económico (salud, educación, retail y administración pública). La ACHS ofreció su apoyo ante las empresas seleccionadas para lograr su colaboración en el estudio.

Se estimó un tamaño muestral de 384 casos con un 95\% de confianza en base a la población ocupada a nivel de la Región Metropolitana en las ramas seleccionadas. Las cuotas por sector se establecieron según los datos de la población ocupada en cada sector económico, en la RM, de acuerdo al siguiente cuadro. (Cuadro 1)

Cuadro 1.

Distribución de las cuotas muestrales por sector económico.

\begin{tabular}{|c|c|c|c|c|c|c|c|c|c|}
\hline & & & & Sectore & & & & & \\
\hline $\begin{array}{l}\text { Ocupados } \\
\text { Región Metropolitana * }\end{array}$ & $\begin{array}{c}\text { Comercio al por mayor } \\
\text { y al por menor }\end{array}$ & $\%$ & $\begin{array}{l}\text { Administración } \\
\text { pública }\end{array}$ & $\%$ & Enseñanza & $\%$ & $\begin{array}{c}\text { Servicios sociales } \\
\text { y de salud }\end{array}$ & $\%$ & Total \\
\hline Total Ocupados & 717.460 & 54,6 & 165.640 & 12,6 & 246.850 & 18,8 & 184.610 & 14,0 & 1.314 .560 \\
\hline
\end{tabular}

Fuente: Nueva Encuesta Nacional de Empleo. INE 2015. 
Una segunda etapa consideraba la selección de casos a entrevistar mediante un procedimiento aleatorio en base a listas de trabajadores/ as facilitadas por las empresas seleccionadas en la primera etapa.

\section{iii. Selección de los casos de la muestra}

Los criterios de aleatoriedad establecidos en el diseño muestral para la selección de empresas y de trabajadores/as no se pudieron cumplir debido a la negativa de muchas empresas a colaborar en el estudio. De este modo, la muestra definitiva estuvo conformada por empresas y trabajadores/as que se ofrecieron a participar voluntariamente en el estudio, por lo cual sus resultados no pueden ser generalizados a todos los/as trabajadores/as de cada sector.

A pesar de las dificultades iniciales, la muestra definitiva con que se trabajó sobrepasó la cuota muestral establecida originalmente debido al interés que suscitó el estudio entre los trabajadores/as y sus dirigencias. La excepción fue el sector del retail, donde no se pudo lograr la cuota estimada debido a que las empresas del sector presentaron más dificultades para colaborar que en los otros sectores. (Cuadro 2)

\section{Cuadro 2.}

Distribución de la muestra definitiva según sector económico.

$\begin{array}{llll}\text { Sector Productivo } & \begin{array}{l}\text { Cuota } \\ \text { Comprometida }\end{array} & \begin{array}{l}\text { Total } \\ \text { cuestionarios } \\ \text { aplicados }\end{array} & \begin{array}{l}\text { Variación } \\ \text { respecto de } \\ \text { lo comprometido }\end{array} \\ \text { Educación } & 72 & 99 & +37,5 \\ \text { Salud } & 54 & 122 & +125,9 \\ \text { Retail } & 210 & 195 & -7,1 \\ \text { Administración Pública } & 48 & 138 & +187,5 \\ \text { Total } & 384 & 554 & +44,3\end{array}$

\section{Resultados}

\section{Resultados Análisis Psicométrico}

a. Análisis factorial

Previo a la exploración de la estructura del instrumento sobre violencia externa, se realizaron pruebas de adecuación muestral. La prueba de Kayser-Meyer-Olkin (KMO) que evalúa la cantidad de varianza explicada por los factores entregó un valor de 0.946 lo que indica que existe suficiente varianza explicada por éstos. Por otro lado la prueba de esfericidad de Bartlett, cuya hipótesis nula es que la matriz de correlaciones es una matriz de identidad (varianza 1 y correlaciones 0) resultó significativa [x2(990) $=15024$.961, $\mathrm{p}=0.000]$, lo que indica que las variables están relacionadas entre sí y resulta razonable realizar el análisis factorial.

A fin de explorar la estructura del instrumento se realizó un análisis factorial mediante un método de extracción por ejes principales. Este método resulta más adecuado que el método de Máxima Verosimilitud al ser más robusto para trabajar variables que contienen únicamente cuatro puntos y no se distribuyen normalmente.

Por otro lado, se utilizó un método de rotación oblicuo "Promax" al considerarse que teóricamente los factores deben estar relacionados entre sí. Además, el número de factores fue determinado por el criterio de un autovalor mayor a 1 . Se suprimieron todas las cargas menores a 0.40 , al ser consideradas como indicativas de items poco confiables. Es posible ver la matriz de cargas factoriales rotadas en la Tabla 1.

Es posible observar que la solución factorial seleccionada constó de siete factores correlacionados entre sí (ver tabla 1) que explicaron un $67,2 \%$ de la varianza. Un primer factor que explicó un
Tabla 1.

Matriz de Cargas Rotadas.

\begin{tabular}{|c|c|c|c|c|c|c|c|}
\hline \multicolumn{8}{|c|}{ Factor } \\
\hline & 1 & 2 & 3 & 4 & 5 & 6 & 7 \\
\hline P4.3 & 1.012 & & & & & & \\
\hline P4.2 & .969 & & & & & & \\
\hline P4.1 & .821 & & & & & & \\
\hline P1.13 & .815 & & & & & & \\
\hline P4.4 & .788 & & & & & & \\
\hline P1.12 & .692 & & & & & & \\
\hline P1.14 & .668 & & & & & & \\
\hline P1.11 & .580 & & & & & & \\
\hline P1.10 & .526 & .465 & & & & & \\
\hline P1.7 & & .867 & & & & & \\
\hline P1.3 & & .844 & & & & & \\
\hline P1.5 & & .810 & & & & & \\
\hline P1.6 & & .796 & & & & & \\
\hline P1.4 & & .780 & & & & & \\
\hline P1.9 & & .741 & & & & & \\
\hline P1.2 & & .661 & & & & & \\
\hline P1.1 & & .646 & & & & & \\
\hline P1.8 & & .642 & & & & & \\
\hline P6.16 & & & .823 & & & & \\
\hline P6.15 & & & .790 & & & & \\
\hline P6.13 & & & .790 & & & & \\
\hline P6.19 & & & .778 & & & & \\
\hline P6.18 & & & .718 & & & & \\
\hline P6.17 & & & .578 & & & & \\
\hline P6.14 & & & .495 & & & & \\
\hline P6.12 & & & .408 & & & & \\
\hline P7.26 & & & & .967 & & & \\
\hline P7.25 & & & & .916 & & & \\
\hline P7.24 & & & & .866 & & & \\
\hline P7.27 & & & & .723 & & & \\
\hline P7.23 & & & & .610 & & & \\
\hline P5.4 & & & & & .835 & & \\
\hline P5.3 & & & & & .807 & & \\
\hline P5.2 & & & & & .742 & & \\
\hline P5.5 & & & & & .469 & & \\
\hline P5.6 & & & & & .438 & & \\
\hline P5.1 & & & & & .405 & & \\
\hline P5.8 & & & & & & .874 & \\
\hline P5.9 & & & & & & .745 & \\
\hline P5.11 & & & & & & .511 & \\
\hline P5.7 & & & & & & .487 & \\
\hline P5.10 & & & & & & & \\
\hline P7.21 & & & & & & & .802 \\
\hline P7.22 & & & & & & & .676 \\
\hline P7.20 & & & & & & & .618 \\
\hline
\end{tabular}

34,1\% de la varianza recogió aquellos ítems de la Pregunta 1 que representan manifestaciones graves de violencia, incluyendo la violencia física por parte de los usuarios, además de los ítems de la Pregunta 4 que incluyen la violencia ejercida por delincuentes. Este factor, entonces, es capaz de recoger las manifestaciones de violencia más asociadas al riesgo de la integridad física. Un segundo factor que explicó un 12,3\% de la varianza recogió aquellas manifestaciones de violencia no física por parte de los usuarios y que no implican un riesgo inminente a la integridad física del/a trabajador/a. El tercer factor explicó un 7,7\% de la varianza y recogió todas las causas de violencia que tienen que ver con el usuario, mientras que el cuarto factor que explicó un 4,3\% de la varianza recogió las causas de violencia que tienen que ver con las 
capacidades laborales del trabajador. El quinto factor explicó un $3,6 \%$ de la varianza y recoge las causas de violencia asociadas a la logística del lugar de trabajo, mientras que el sexto factor explicó un 2,7\% de la varianza y recoge las causas de violencia asociadas al apoyo que brinda el lugar de trabajo a sus trabajadores/as o funcionarios/as. Finalmente, el séptimo factor explicó un $2,6 \%$ de la varianza y recoge las causas de violencia relacionadas con las características personales del trabajador/a o funcionario/a.

Individualmente, la pregunta 1.10 asociada a las amenazas de violencia física presentó una carga cruzada entre el factor 5 y 6 , mientras que la pregunta 5.10 asociada a que el trabajador no cuenta con información suficiente como causa de violencia no tuvo carga superior a 0.40 en ningún factor, indicando que no es un item que represente de manera confiable alguna de las dimensiones. Se considera tomar ambos ítems con precaución al momento de la utilización del instrumento. El item 4.3 presentó una carga ligeramente mayor a 1, sin embargo, es consistente teóricamente y el mejor representante del factor, lo que puede ser interpretado como un ítem altamente confiable. Estos ítems han sido marcados en negrita en la Tabla 1.

Por otro lado, es posible observar en la tabla 2 las medias por sector de las variables de interés.

Tabla 2.

Medias por sector.

$\begin{array}{lcccccccc}\text { Sector } & \text { Fact1 } & \text { Fact2 } & \text { Fact3 } & \text { Fact4 } & \text { Fact5 } & \text { Fact6 } & \text { Fact7 } & \text { GH012 } \\ \text { Educación } & 1.31 & 1.80 & 2.74 & 1.88 & 2.32 & 2.44 & 2.13 & 2.04 \\ \text { Salud } & 1.44 & 2.15 & 2.84 & 1.70 & 2.67 & 2.74 & 1.90 & 1.73 \\ \text { Retail } & 2.21 & 2.43 & 2.57 & 2.09 & 2.53 & 2.70 & 2.25 & 2.00 \\ \text { Adm. Pública } & 1.34 & 2.12 & 2.82 & 1.62 & 2.60 & 2.83 & 2.02 & 2.03\end{array}$

Para realizar un "benchmarking” entre los distintos sectores respecto a la intensidad de la violencia y sus causas, se realizó una prueba de ANOVA de una vía con los factores como variable dependiente y los sectores como factores, con un valor p crítico de 0.05 .

En el caso del primer factor (items de la pregunta1 y de la pregunta 4), que representa manifestaciones graves de violencia, se encontró un efecto significativo del sector en la intensidad de la violencia física $[\mathrm{F}(1,546)=7.81, \mathrm{p}=.005]$, el que se evaluó con más detalle utilizando contrastes no planificados con un método de ajuste Bonferroni, el que indicó que Retail presenta un nivel significativamente mayor de intensidad de la violencia grave que todos los demás sectores, los que no se diferenciaron entre ellos. (Figura 1)

\section{Figura 1.}

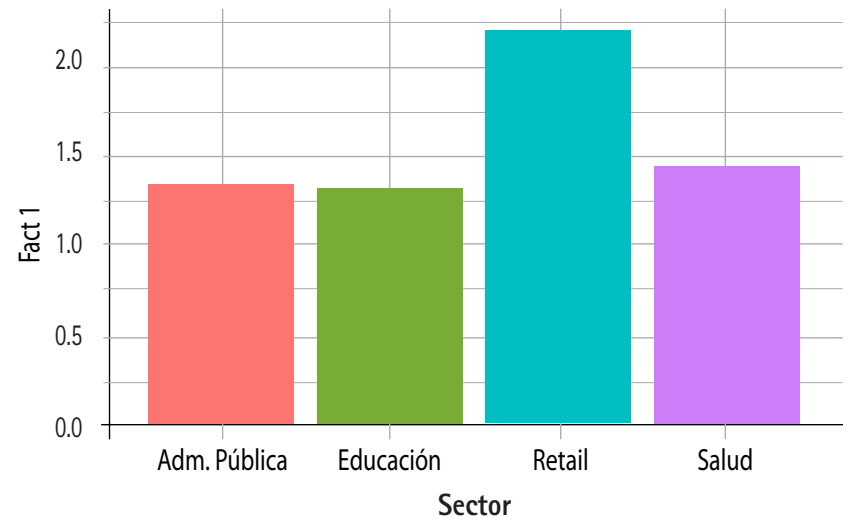

En el caso del segundo factor (manifestaciones de violencia no física por parte de los usuarios), se encontró un efecto significativo del sector en la intensidad de la violencia no física $[\mathrm{F}(1,545)=12.51$, $\mathrm{p}=0.000$, el que se evaluó con más detalle utilizando contrastes no planificados con un método de ajuste Bonferroni, el que indicó que retail presenta un nivel significativamente mayor de intensidad de la violencia no física que todos los demás sectores. Por otro lado, educación presentó un nivel significativamente menor de intensidad de la violencia no física que los demás sectores. (Figura 2)

\section{Figura 2.}

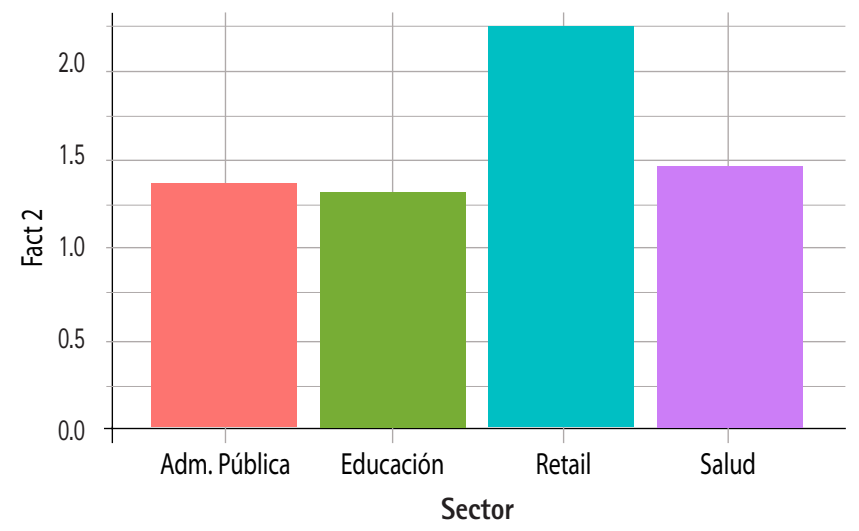

En el caso del tercer y cuarto factor, indicativos de las causas de violencia relativas a los usuarios $[\mathrm{F}(1,548)=0.057, \mathrm{p}=.811]$ y a las características del trabajador $[\mathrm{F}(1,541)=0.84, \mathrm{p}=0.36]$ no se encontraron diferencias significativas según el sector.

En el caso del quinto factor (causas de la violencia asociadas al lugar de trabajo), se encontró un efecto significativo del sector en la intensidad de las causas de la violencia asociadas a la logística del lugar de trabajo $[\mathrm{F}(1,551)=4.575, \mathrm{p}=0.033]$, el que se evaluó en más detalle utilizando contrastes no planificados con un método de ajuste Bonferroni, el que indicó que educación presenta un nivel significativamente menor de esta intensidad que salud y administración pública, pero no se diferenció significativamente del sector retail. (Figura 3)

En el caso del sexto factor (apoyo del lugar de trabajo a trabajadores/ as o funcionarios/as), se encontró un efecto significativo del sector en la intensidad de las causas de la violencia asociadas al apoyo que

Figura 3.

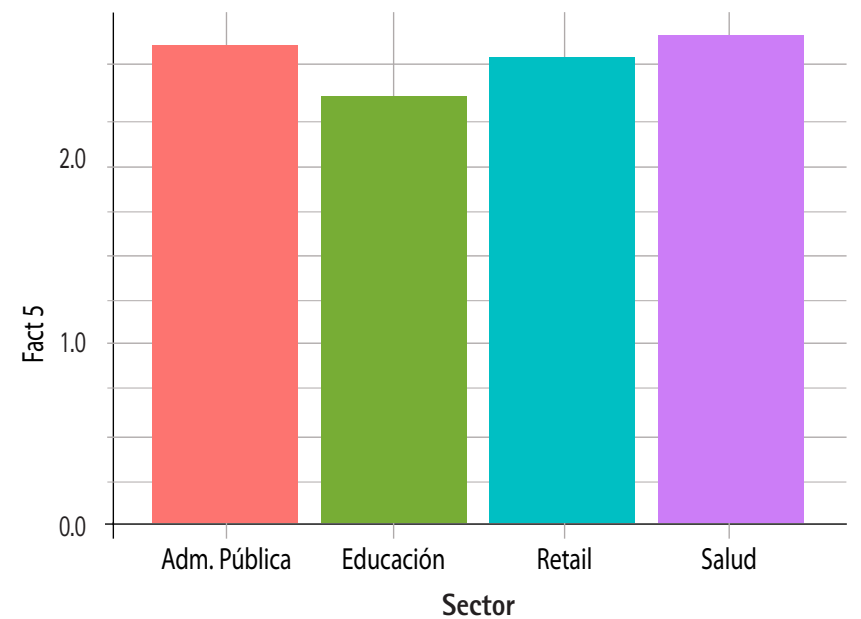


les brinda el lugar de trabajo $[\mathrm{F}(1,551)=10.95, \mathrm{p}=0.001]$, el que se evaluó con más detalle utilizando contrastes no planificados con un método de ajuste Bonferroni, el que indicó que educación presenta un nivel significativamente menor de esta intensidad que todos los demás sectores, los que no se diferenciaron entre sí. (Figura 4)

Figura 4.

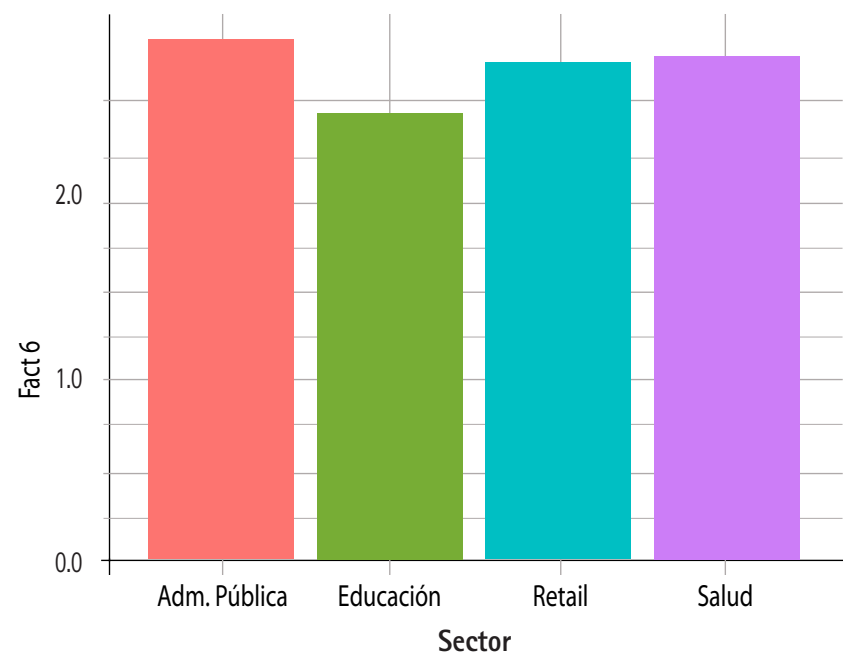

En el caso del séptimo factor, no se encontró un efecto significativo del sector en las causas de violencia relativas a las características personales del trabajador $[\mathrm{F}(1,542)=0.187, \mathrm{p}=0.666]$.

Pruebas de validez convergente: análisis de correlaciones entre los distintos factores y la medida de salud (GHQ-12)

Se computó una matriz de correlaciones entre los factores seleccionados y la medida de salud general (GHQ-12). Los valores de intensidad de la correlación (r) pueden ser observados en la diagonal inferior, mientras que los valores de probabilidad (p) ajustados por comparaciones múltiples mediante un método de Holm-Bonferroni pueden ser observados en la diagonal superior. (Tabla 3)

Tabla 3.

Matriz de correlaciones entre los factores y salud general.

$\begin{array}{lcccccccc} & \text { Fact1 } & \text { Fact2 } & \text { Fact3 } & \text { Fact4 } & \text { Fact5 } & \text { Fact6 } & \text { Fact7 } & \text { GHO12 } \\ \text { Fact1 } & 1 & 0 & 0 & 0 & 0 & 0 & 0 & 0.01 \\ \text { Fact2 } & 0.65 & 1 & 0 & 0 & 0 & 0 & 0 & 0 \\ \text { Fact3 } & 0.22 & 0.53 & 1 & 0 & 0 & 0 & 0 & 0.01 \\ \text { Fact4 } & 0.41 & 0.44 & 0.38 & 1 & 0 & 0 & 0 & 0.01 \\ \text { Fact5 } & 0.24 & 0.4 & 0.57 & 0.42 & 1 & 0 & 0 & 0.01 \\ \text { Fact6 } & 0.17 & 0.36 & 0.51 & 0.35 & 0.62 & 1 & 0 & 0 \\ \text { Fact7 } & 0.32 & 0.34 & 0.41 & 0.59 & 0.44 & 0.35 & 1 & 0 \\ \text { GH012 } & 0.11 & 0.19 & 0.13 & 0.14 & 0.13 & 0.17 & 0.15 & 1\end{array}$

Nota: La diagonal superior indica los valores de probabilidad ( $p$ ) mientras que la diagonal inferior indica la intensidad de las correlaciones.

Es posible observar que todos los factores se encuentran correlacionados entre sí, mientras que las correlaciones entre los factores y la salud general (mental) son de una intensidad baja. Este nos parece un resultado esperable ya que la violencia externa podría ser considerada una variable distal en la cadena causal hacia los problemas de salud mental. Se recomienda incluir para futuros estudios posibles variables moderadoras o mediadoras de esta relación.

Estandarización del instrumento: cálculo de puntajes, punto de corte o baremos sobre el grado de exposición a violencia externa.
El punto de corte se realizó mediante una curva ROC utilizando un método de Youden que busca la maximización de la especificidad y sensibilidad del baremo.

Se calcularon los puntajes para establecer la presencia de violencia y de sus causas, tomando como referencia de ausencia el valor 1 (Casi nunca, nunca). Se recodificaron las variables de la siguiente forma: $1=0,2=1,3=1,4=1$. Los puntajes de la variable recodificada fueron sumados para crear el índice de presencia de violencia externa.

Para calcular la presencia de violencia se tomaron en cuenta únicamente los ítems de las preguntas 1 y 4 , y se tomaron como valores de referencia aquellos que representan -1 (3.87), 0 (9.49), 1 (15.11) desviaciones estándar de la media. No se tomó en cuenta el valor de +-2 desviaciones estándar dado que la distribución no permitió un valor real en este punto al contar con poca dispersión. Los puntajes brutos asociados se encuentran entre paréntesis. La escala cuenta con un rango de 0 a 18 puntos.

Para calcular la presencia de causas de violencia se tomaron en cuenta únicamente los ítems de las preguntas 5, 6 y 7, y se tomaron como valores de referencia aquellos que representan -2 (9.84), -1 (15.8) y 0 (20.32) desviaciones estándar de la media. No se tomó en cuenta el valor de +1 desviación estándar ya que su puntaje aproximado se encontró fuera del rango (26 puntos) al contar con una distribución con asimetría negativa. Los puntajes brutos asociados se encuentran entre paréntesis. La escala cuenta con un rango de 0 a 26 puntos.

Para calcular la intensidad de la violencia y de sus causas se sumaron los puntajes brutos de las escalas.

En el caso de la intensidad de la violencia se tomaron en cuenta únicamente los items de las preguntas $1 \mathrm{y} 4$, y se tomaron como valores de referencia aquellos que representan -2 (6.18) -1 (20.02), 0 (33.86), 1 (47.7) y 2 (61.54) desviaciones estándar de la media. No se tomó en cuenta el valor de -2 desviaciones estándar dado que el puntaje mínimo en la escala es de 18 puntos. Los puntajes brutos asociados se encuentran entre paréntesis. La escala cuenta con un rango de 18 a 72 puntos.

En el caso de la intensidad de las causas de la violencia se tomaron en cuenta únicamente los items de las preguntas 5, 6 y 7, y se tomaron como valores de referencia aquellos que representan -2 (32.91) -1 (47.69), 0 (62.47), 1 (77.25) y 2 (92.03) desviaciones estándar de la media. No se tomó en cuenta el valor de -2 desviaciones estándar dado que el puntaje mínimo en la escala es de 18 puntos. Los puntajes brutos asociados se encuentran entre paréntesis. La escala cuenta con un rango de 26 a 104 puntos.

Fórmula de cálculo de presencia e intensidad de violencia y de causas de violencia

La PRESENCIA de violencia externa se obtiene simplemente contando cuantas respuestas de las 18 posibles de las P.1 y P.4 respondieron "siempre", "a menudo" o "pocas veces". La presencia de la violencia externa en el/la trabajador/a estudiado/a será considerada:

Nula $=0$ items positivos.

Muy baja = Entre 1 y 3 items positivos.

Baja $=$ Entre 4 y 9 ítems positivos.

Moderada $=$ Entre 10 y 14 items positivos.

Alta $=$ Entre $15 \mathrm{y} 18$ items positivos.

La INTENSIDAD de la violencia se cuantifica sumando la frecuencia de las situaciones de violencia externa señaladas por los sujetos y se obtiene otorgando a las respuestas dadas por el sujeto el siguiente puntaje: 


$\begin{array}{ll}\text { Siempre } & =4 \\ \text { A menudo } & =3 \\ \text { Pocas Veces } & =2 \\ \text { Casi nunca, Nunca } & =1\end{array}$

Por ejemplo, si la persona encuestada señala haber estado expuesta a 5 situaciones de violencia de las 18 que figuran en el instrumento, es decir aquellas en las que respondió "siempre" o "a menudo" o "pocas veces", su puntaje es 5 y la presencia de violencia es baja en este caso. Si de las 18 situaciones, 2 ocurrieron "siempre", 2 ocurrieron "a menudo", 1 ocurrió "pocas veces" y 13 ocurrieron "nunca o casi nunca", la intensidad de la violencia será de $2 \times 4+2 \times 3+1 \times 2+$ $13 \times 1=29$ puntos. En este caso la intensidad es baja.

La INTENSIDAD de la Violencia en el/la trabajador/a estudiado/a se considerará conforme a la siguiente tabla:
Alta Intensidad (ocurre siempre)
$=62$ a 72.
Intensidad media-alta (ocurre a menudo)
Mediana Intensidad (ocurre pocas veces)
Baja Intensidad (ocurre casi nunca)
$=48$ a 61 .
$=34 \mathrm{a} 47$.
$=18$ a 33 .

La PRESENCIA de causas de violencia externa se obtiene simplemente contando cuantas respuestas de las 26 posibles de las P.5, P.6, y P.7 presentaron un valor distinto de 1 (Casi nunca, nunca).

La presencia de las causas de violencia externa será considerada:

Nula $=0$ ítems positivos.

Muy baja = Entre 1 y 9 items positivos.

Baja $=$ Entre 10 y 15 items positivos.

Moderada $=$ Entre 16 y 19 items positivos.

Alta $=$ Entre $20 \mathrm{y} 26$ ítems positivos.

La INTENSIDAD de las causas de violencia se cuantifica sumando la frecuencia de las situaciones de violencia externa señaladas por los sujetos y se obtiene otorgando a las respuestas dadas por el sujeto el siguiente puntaje:

$\begin{array}{ll}\text { Siempre } & =4 \\ \text { A menudo } & =3 \\ \text { Pocas Veces } & =2 \\ \text { Casi nunca, Nunca } & =1\end{array}$

La INTENSIDAD de las causas de Violencia se calcula siguiendo igual procedimiento que en el caso de INTENSIDAD de la violencia y se considera conforme a la siguiente tabla:

Muy alta intensidad $=92$ a 104

Alta intensidad $\quad=\quad 77$ a 91

Intensidad Media-Alta $=62$ a 76

Mediana intensidad $\quad=\quad 48$ a 61

Baja intensidad $\quad=\quad 27$ a 47

\section{RESULTADOS}

\section{Cómo se vive la violencia externa en el trabajo}

Percepciones de trabajadores y trabajadoras de cuatro sectores: Educación, salud, retail y administración pública

Los resultados revelaron que la violencia externa se vive cotidianamente en los lugares de trabajo. Con mayor o menor frecuencia, la experimentan prácticamente todos y todas los trabajadores/as de los cuatro sectores estudiados. Se constatan, sin embargo, diferencias y similitudes entre ellos.
Al comparar sectores, se observa que el Retail presenta un nivel significativamente mayor de presencia e intensidad de la violencia que todos los demás sectores, los que no se diferenciaron entre ellos y un nivel significativamente mayor de intensidad de la violencia. En general, los estudios sobre este problema han destacado que uno de los sectores mayormente expuestos a diversas formas de violencia es el sector de la salud, resultado que no se verifica en este estudio. La presencia e intensidad de la violencia en este sector muestra valores inferiores pero relativamente cercanos a los que se constatan en el sector de la administración pública, lo cual nos parece importante destacar, en especial si se tiene en consideración que estamos hablando de instituciones públicas puesto que los establecimientos de salud en los cuales se aplicó el instrumento pertenecen al sistema público. El sector de la educación es el que presenta menor presencia e intensidad de violencia externa.

En relación a las formas que asume la violencia se observan diferencias relevantes entre sectores. En concordancia con una mayor presencia e intensidad, los/as trabajadores del Retail señalan estar expuestos a 12 de las 14 situaciones descritas en el instrumento, versus los otros sectores estudiados en los que las respuestas se concentran en un número bastante menor de situaciones.

$\mathrm{Al}$ comparar las percepciones de hombres y mujeres en estos aspectos, se observó una diferencia significativa entre hombres $(\mathrm{M}=1.89)$ y mujeres $(\mathrm{M}=1.46)$ en la intensidad de la violencia física (Factor 1); $\mathrm{t}(461)=6.34, \mathrm{p}=.000$. También se observó una diferencia significativa entre hombres $(\mathrm{M}=2.63)$ y mujeres $(\mathrm{M}=2.80)$ en las causas de violencia que tienen que ver con el usuario (Factor 2); $t(505)=3.04, p=.002$. Por otro lado, no se observaron diferencias significativas en los demás factores entre hombres y mujeres.

Llama la atención que las mujeres experimentan menor presencia e intensidad de violencia que los hombres, hallazgo que marca una tendencia contraria a resultados de otros estudios. Asimismo, se observan diferencias entre ellos relativas a las situaciones de violencia que enfrentan, destacándose una menor presencia de violencia física en las mujeres en todos los sectores y mayor presencia de violencia psicológica ("miradas o gestos intimidatorios o amenazantes", "reacciones de enojo exageradas”, "gritos”, "insultos, garabatos y groserías" y "trato prepotente").

En relación a los factores de riego, un hallazgo relevante es que los/ as trabajadores/as de todos los sectores, de ambos sexos, señalan que estos derivan principalmente de condiciones del lugar de trabajo y de los/as clientes o usuarios/as y en mucho menor medida de sus propias características. La "edad" de los/as trabajadores/as no constituye un factor de riesgo en tanto no se asociada ni a presencia ni intensidad de la violencia, es decir no hay grupos de edad más expuestos a violencia que otros, ni en hombres ni en mujeres. Asimismo, tampoco son factores de riesgo la calidad del empleo (tipo de contrato), ni la antigüedad en el mismo.

Al comparar sectores, es importante destacar que nuevamente se constatan similitudes entre las percepciones de los/as trabajadores/as del retail y de la administración pública. Ambos/as coinciden en considerar, en proporciones mucho más alta que los otros dos sectores, que los factores causales de la violencia externa son algunas condiciones del lugar de trabajo ("personal insuficiente para atender a los/as usuarios/as", "demora en la atención/largos tiempos de espera”, "escaso tiempo para atender necesidades del/a usuario/a", "falta de apoyo de la empresa o institución para enfrentar problemas con los/as usuarios/as" y "falta de protección). Asimismo, proporciones mayores de trabajadores/as de la salud que de los otros sectores opinan que las características o formas de comportamiento 
de los/as usuarios/as son las causantes de la violencia, opiniones en buena parte compartidas por los/as trabajadores/as de la administración pública.

Los grupos más expuestos a situaciones de violencia externa son los trabajadores y trabajadoras del retail, seguidos por aquellos/as que se desempeñan en el sector de la salud y de la administración pública. A la pregunta de los factores de riesgo asociados a características de los y las trabajadores/as, un 34,6\% de las mujeres y un 22,6\% de los hombres respondió el "ser mujer", un 24\% respondió "ser extranjero", un 22,5\% respondió "pertenecer a una etnia o rasa en particular" y un $24 \%$ respondió la "orientación sexual" del o la trabajadora. De aquí se puede inferir entonces que pertenecer a uno de estos grupos sitúa a las personas en situación de riesgo de experimentar violencia externa.

Las víctimas de violencia laboral externa reportan mayor posibilidad de sufrir problemas de salud mental versus los/as trabajadores/as que no han sido víctimas en tanto hombres y mujeres que reportaron haber sido víctimas violencia externa Alta o Moderada fueron quienes reportaron mayores indicios de presencia de psicopatología. Asimismo, trabajadores y trabajadoras que experimentaron una intensidad de violencia Alta y Media Alta presentaron mayores indicios de presencia de psicopatología en comparación con aquellos/as que no habian estado expuestos a violencia externa (aproximadamente $20 \%$ versus un $10 \%$ ).

La tercera hipótesis del estudio no se confirma en tanto, como ya hemos señalado, las mujeres experimentaron menor presencia e intensidad de la violencia en todos los sectores, y los indicativos de presencia de psicopatología muestran muy pocas diferencia entre hombres y mujeres.

\section{DISCUSION DE RESULTADOS}

Todas las pruebas estadísticas aplicadas demostraron que el instrumento elaborado cumple con sus objetivos de medición, y está construido de manera tal que entrega un diagnóstico de la presencia e intensidad de violencia externa percibida, con diferencias entre trabajadores y trabajadoras de atención directa a público, es decir, es capaz de discriminar diferenciadamente la exposición al factor de riesgo y sus causas.

A juicio del equipo, realizar análisis factorial en dos momentos fue una decisión acertada, ya que permitió ajustar algunas preguntas que, si bien la evidencia internacional las señalaban como determinantes, para el caso de nuestro país y nuestra cultura laboral, no tenían igual peso en términos de causalidad de la violencia externa. El proceso también permitió hacer ajustes semánticos al cuestionario.

En términos de representatividad, el instrumento cumple con los requisitos para ser utilizado en diversos sectores productivos, dado que cuenta con una escala capaz de discriminar diferentes situaciones de presencia e intensidad de la violencia externa.

Respecto a las asociaciones entre exposición a violencia externa y problemas de salud general medidos a través del GHQ 12 (Global Health Questionnaire) el estudio reveló correlaciones de baja intensidad, lo cual es esperable si se considera que la violencia externa es una variable distal de la cadena causal de los problemas de salud general. Se recomiendo incluir para futuros estudios posibles variables moderadoras o mediadoras de esta relación. 


\section{REFERENCES}

1. Hoel $\mathrm{H}$, Einarsen $\mathrm{S}$, Cooper CL. Bullying and emotional abuse in the workplace. International perspective in research and practice. London: Taylor $\&$ Francis; 2003.

2. Di Martino $V_{\text {, Hoel }} \mathrm{H}_{\text {, Cooper }} \mathrm{CL}$. Preventing violence and harassment in the workplace. Luxembourg: European Foundation for the Improvement of Living and Working Conditions; 2003.

3. Di Martino V. Workplace violence in the health sector. Country case studies Brazil, Bulgaria, Lebanon, Portugal, South Africa, Thailand, plus an additional Australian study; Synthesis report [on line]. [Geneva]: ILO/ICN/WHO/PSI ; 2002 [cited 2018 Apr]. Available from: http://www.who.int/violence_injury_ prevention/injury/en/WVsynthesisreport.pdf.

4. Chappell D, Di Martino V. Violence at Work. 3a ed. Geneva: International Labour Office; 2006.

5. Pérez Bilbao J, Nogareda Cuixart C. NTP 489: Violencia en el lugar de trabajo. Madrid: Instituto Nacional de Higiene y Seguridad en el Trabajo; 1998.

6. Eurofound. Fifth European Working Conditions Survey [on line]. Luxemburg: Publications Office of the European Union; 2012 [ cited 2018 Apr]. Available from: https://www.eurofound.europa.eu/sites/default/files/ef_publication/ field_ef_document/ef1182en.pdf

7. Vézina $M$, Cloutier $E$, Stock $S$, Lippel $K$, Fortin $E$, Delisle $A$, et al. Enquête québécoise sur des conditions de travail, d'emploi et de santé et de sécurité du travail (EOCOTESST). Québec : Institut national de santé publique du Québec-Institut de la statistique du Québec-Institut de recherche RobertSauv; 2011.

8. Einarsen S, Skogstad A. Bullying at work: Epidemiological Findings in Public and Private Organizations Eur J Work Organ Psy [on line]. 1996 [cited 2018 May]; 5(2):185-201. Available from: http://dx.doi.org/10.1080/13594329 608414854

9. European Agency for Safety and Health at Work. European Risk Observatory Report. Workplace Violence and Harassement: a European picture. Luxembourg: Publications Office of the European Union; 2010.

10. Leather P. Workplace violence: Scope, definition and global context. In: Cooper CL, Swanson N. Workplace violence in the health sector; State of the Art. Geneva: WHO; 2002. p. 3-18.

11. Paravic T, Valenzuela S, Burgos M. Violencia Percibida por Trabajadores de Atención Primaria de Salud. Cienc enferm. 2004; 10(2):53-65.
12. Díaz $X$, Mauro A, Toro JP, Villarroel C, Campos D. Validación del inventario de violencia y acoso psicológico en el trabajo -IVAPT-PANDO- en tres ámbitos laborales chilenos. Cienc Trab [en línea]. 2015 [citado may 2018]; 17(52):7-14. Disponible en: http://www.cienciaytrabajo.cl/cyt/Paginas/ Validacion-del-Inventario-de-Violencia-y-Acoso-Psicologico-en-elTrabajo.aspx

13. Centro de Estudios de la Mujer-Universidad de Ottawa. Proyecto Araucaria [sitio web]. Santiago de Chile: Centro de Estudios de la Mujer; c2007-2012 [consultado abr 2018]. Disponible en: www.proyectoaraucaria.cl.

14. Eurofound. Fourth European Survey on Working Conditions. Luxembourg: Office for Official Publications of the European Communities; 2007 [cited 2018 Apr]. Available from: https://www.eurofound.europa.eu/sites/default/ files/ef_publication/field_ef_document/ef0698en.pdf

15. Cooper CL, Swanson N. Workplace violence in the health sector; State of the Art. Geneva: International Council of Nurses; 2002.

16. European Agency for Safety and Health at Work. Assessing and trackling external violence at work. Case Studies [on line]. Bilbao: EU- OSHA ; 2009 [cited 2018 May]. Available from: https://osha.europa.eu/en/tools-andpublications/publications/assessing-and-tackling-external-violence-atwork

17. Alvesson M, Billing, YD. Understanding Gender and Organization. London: SAGE; 1997.

18. Krieger N, Waterman PD, Hartman C, Bates L, Stoddard AM, Quinn M et al. Social hazards on the job: workplace abuse, sexual harassment, and racial discrimination- a study of black, latino, and white low-income women and men workers in the United States. Int J Health Serv. 2006; 36(1):51-85.

19. OIT-OMS-CIE-ISP. Directrices marco para afrontar la violencia laboral en el sector de la salud [en línea]. Ginebra: Programa Conjunto sobre violencia laboral en el sector de la salud; 2002 [citado mar 2018]. Disponible en: http://apps.who.int/iris/bitstream/handle/10665/44072/922313 4463_spa.pdf;jsessionid=2850E5EA3F175214394177E2CB24A68A?sequ ence $=1$

20. Rodríguez de la Pinta ML, Maestre M, Borda J. Violencia externa en el trabajo en el sector Servicios. Consideraciones generales, notificación y protocolos de actuación. Med segur trab [en línea]. 2011 [citado abr 2018]; 57(supl.1):53-71. Disponible en: http://dx.doi.org/10.4321/S0465-546X 2011000500005 